\title{
DESARROLLO Y PRODUCCIÓN DE ESQUEJES DE TALLO JUVENIL EN PAPA OBTENIDOS EN CUATRO PARTES DIFERENTES DE LA MISMA PLANTA
}

\section{JUAN AGUILAR; JULIO MOLINA; CESAR VITTORELLI*}

\section{RESUMEN}

Para llevar a cabo la presente investigación se usaron esquejes de tallo juvenil de la variedad Mariva (Solanum andigena $\mathrm{x}$ Solanum tuberosum) con el fin de evaluar el desarrollo de la planta al trasplante, a los 30 y 60 días, así como también el número y el peso de los tubérculos y la precocidad. Los esquejes se obtuvieron de 4 diferentes partes de la planta: Parte Apical (PA); primera hoja o nudo como parte media superior (PMS); segunda y tercera hoja o nudo como parte media inferior (PMI) y la cuarta hoja o nudo como parte basal (PB). Los resultados indican que al momento del transplante y a los 30 días no hay mayores diferencias en cuanto a la altura de las plantas, pero sí a los 60 días, notándose un mayor desarrollo de las plantas provenientes de PMS y PMI. Respecto al número de tubérculos, la PA tiene una diferencia de aproximadamente 1 tubérculo más que el resto de tratamientos. Sin embargo, en el peso total no hay mayores diferencias entre los tratamientos. El tamaño de los tubérculos con referencia al peso promedio, señala un tamaño adecuado para su siembra en el campo. Referente a la precocidad, PA mostró ser más precoz que los otros tratamientos con 3 semanas de anticipación.

Palabras Claves Adicionales: Parte apical, primera hoja o nudo de parte media superior; segunda y tercera hoja o nudo de parte media inferior y cuarta hoja o nudo de parte basal.

\section{ABSTRACT \\ Development and Yield of Potato Cutting from Young Stems obtained from four Different Plant Parts.}

Single node stem cutting of the variety Mariva (Solanum andigena $\mathrm{x}$ Solanum tuberosum) were used to evaluate development from plant to transplant, 30 and 60 days after transplanting, number and weight of tubers, and earliness. Stem cutting from four different plant parts were obtained: apical (PA); first leaf or Knot as upper medium part (PMS); second and third leaf or knot as lover medium part (PMI), and fourth

\footnotetext{
* Ingeniero Agrónomo, Especialista en producción de semilla básica; Ingeniero Agrónomo, Encargado de la Producción de Semilla, La Molina e Ingeniero Agrónomo Líder del Proyecto Producción de Semilla básica. Programa Nacional de Papa, Estación Experimental "La Molina" INIPA, Ministerio de Agricultura y Cría, Lima, Perú.
} 
leaf or knot as basal part (PB) . Results show that during the first 30 days from the time of transplanting there are no significant differences as to plant height, but there surely derived are after 60 days when greater development of plants from PMS and PMI parts is registered. As far as the number of tubers is concerned, the apical part (PA) produced aproximately 1 more tuber than the rest of the treatments. However, there were no differences in total weight between treaments. The size of the tubers according to their average weight indicates they have the right size to be used as planting material in the field. The PA showed to be earlier than the other treatments, by 3 weeks.

Additional Index Words: apical, upper medium part, lover medium part, basal part, knot.

Una de las principales metas en un programa de semilla básica de papa es obtener la mayor cantidad de tubérculos pre-básicos, en un corto período de tiempo para ser multiplicados posteriormente en condiciones de campo.

Los centros de producción reciben o producen material libre de enfermedades para ser multiplicado en los invernaderos. Dicho material puede ser en forma de plántulas in vitro o tuberculillos y su incremento se basa en el uso combinado de las técnicas de multiplicación acelerada, desarrollada por el CIP. El incremento se efectúa tanto en macetas para plantas madres como en camas de producción de tuberculillos.

En el presente trabajo se midió la eficiencia de los esquejes de tallo juvenil, obtenidos por la técnica de multiplicación rápida descrita por Bryan (2) según su ubicación en la planta. Este estudio se realizó en vista de que en campañas anteriores se observó desuniformidad en la maduración de las plantas provenientes de esquejes.

\section{MATERIALES Y MÉTODOS}

La presente investigación se llevó a cabo en los invernaderos del Programa Nacional de Papa de la Estación Experimental de La Molina, durante los meses de Mayo, Junio, Julio y Agosto de 1985 correspondiendo a la época normal de siembra en la costa peruana.

Las temperaturas promedio durante el período del estudio fueron: máxima $20.0^{\circ} \mathrm{C}$ y mínima $13.2^{\circ} \mathrm{C}$.

Los esquejes se obtuvieron de cuatro partes de la planta como se muestra en la Figura. 


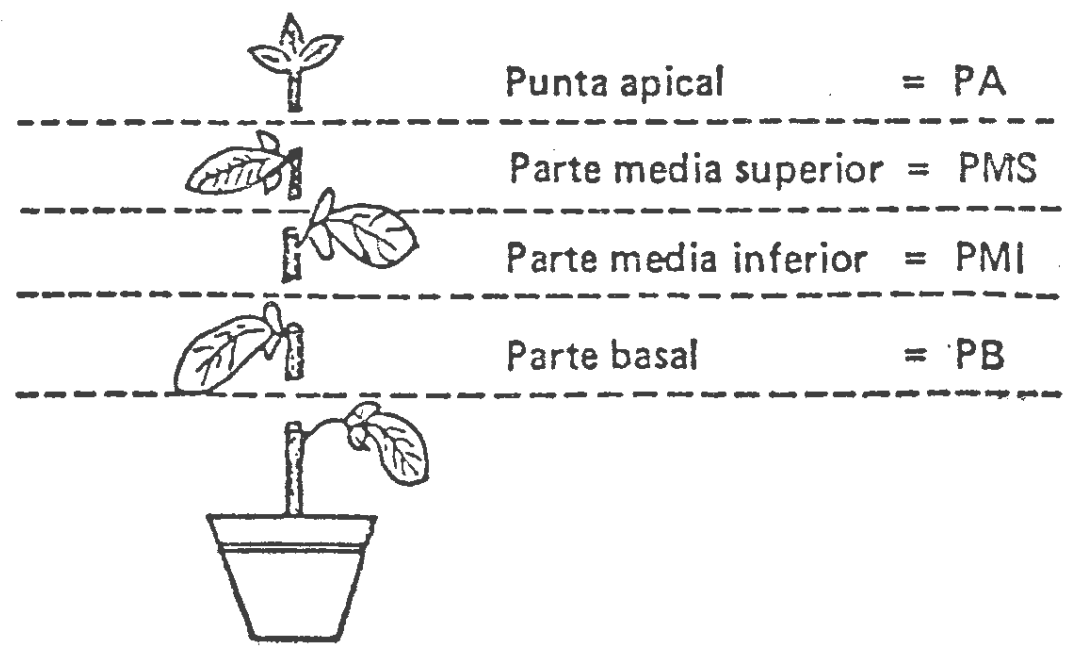

Esquejes de tallo juvenil

El diseño empleado fue el de bloques completos al azar con 4 repeticiones. Se utilizó una mezcla de suelo 2:1:1 (musgo, suelo, arena). El suelo se desinfectó con Bromuro de Metilo aplicándose una dosis de 1.5 libras $/ \mathrm{m}^{3}$. Para el transplante de los esquejes se emplearon 120 macetas previamente lavadas y desinfectadas con Hipoclorito de Calcio al $10 \%$ y cada una de ellas fue llenada hasta la mitad (1 kg de suelo) para poder efectuar los aporques respectivos según el desarrollo de las plántulas e incentivar la tuberización.

La fertilización fue de: N 160 ppm; P205 160 ppm y K20 120 ppm.

Los elementos menores se aplicaron en forma de aspersión foliar cuando las plantas tuvieron $\pm 15 \mathrm{~cm}$ de altura. Se utilizó como producto comercial el Fetrilon Combi, (elementos menores en forma de quelatos) para prevenir las deficiencias que pudieran presentarse. Con un desarrollo de las plantas, aproximadamente a los $6 \mathrm{~cm}$, se aplicó como fungicida específico el Pentacloro Nitrobenceno (PCNB) en dosis del $3 \%$, para prevenir ataques de hongos de suelo, además Temik 15 G como nematicida al substrato en dosis de 35 g para 120 kg de suelo.

Las plantas madres con un promedio de $20 \mathrm{~cm}$ de altura, aproximadamente a los 25 días, se sometieron al corte de esquejes de tallo juvenil: primero se cortó la parte apical (PA) luego la primera hoja (PMS), después la segunda y tercera hojas (PMI) y por último la hoja basal (PB), cada una con su respectiva yema axilar, 
colocándolos en una bandeja previamente lavada y desinfectada, sobre un papel toalla húmedo en la base para evitar la deshidratación, Bryan (1).

Los esquejes de tallo juvenil fueron separados en las respectivas categorías (PA, PMS, PMI, y PB), y tratados con una hormona de enraizamiento, fórmula CIP (2).

Los esquejes tratados se colocaron en arena tamizada $2 \mathrm{~mm}$, lavada y desinfectada para su enraizamiento. Después de 15 días cuando presentaron raíces suficientemente robustas, se efectuó el trasplante, sembrando dos esquejes por maceta.

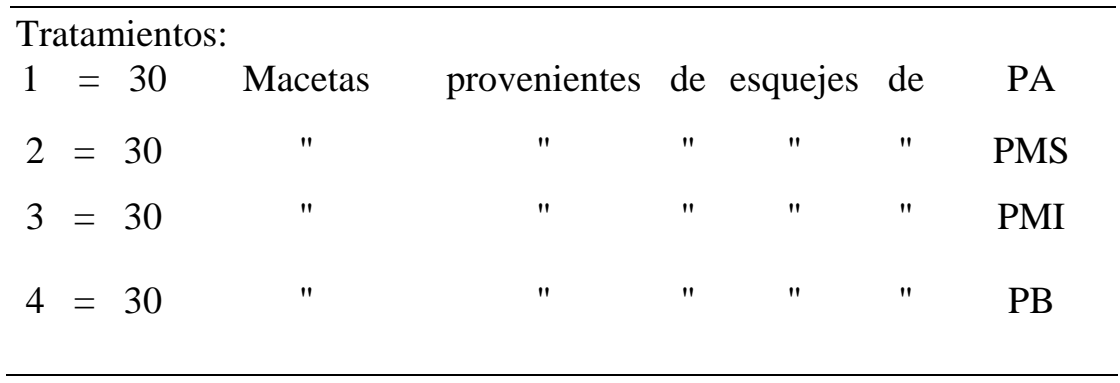

\section{RESULTADOS}

Altura de Planta

Se hicieron tres evaluaciones de altura de planta: al momento del trasplante, a los 30 y 60 días (Tabla 1 y Figura 1). Al momento del trasplante se observó diferencia altamente significativa entre los tratamientos, determinándose el orden decreciente siguiente: PMI, PA, PB y PMS. esqueje

Tabla 1. Evaluaciones de altura de planta según el tipo de

\begin{tabular}{|c|c|c|c|}
\hline & $\begin{array}{l}\text { Al trasplante } \\
(\mathrm{cm})\end{array}$ & $\begin{array}{l}30 \text { días } \\
(\mathrm{cm})\end{array}$ & $\begin{array}{l}60 \text { días } \\
(\mathrm{cm})\end{array}$ \\
\hline Parte apical & 4.7 (a) & 26.7 (b) & 29.4 (b) \\
\hline Parte media superior & $3.5(\mathrm{~b})$ & 29.7 (a) & $40.2(a)$ \\
\hline Parte media inferior & 5.2 (a) & $29.6(a b)$ & 40.5 (a) \\
\hline Parte basal & 3.6 (b) & $27.1(\mathrm{ab})$ & 35.8 (a) \\
\hline $\mathrm{n}=30$ & $34.4 \%$ & $C V=15.5 \%$ & $C V=19.20$ \\
\hline
\end{tabular}




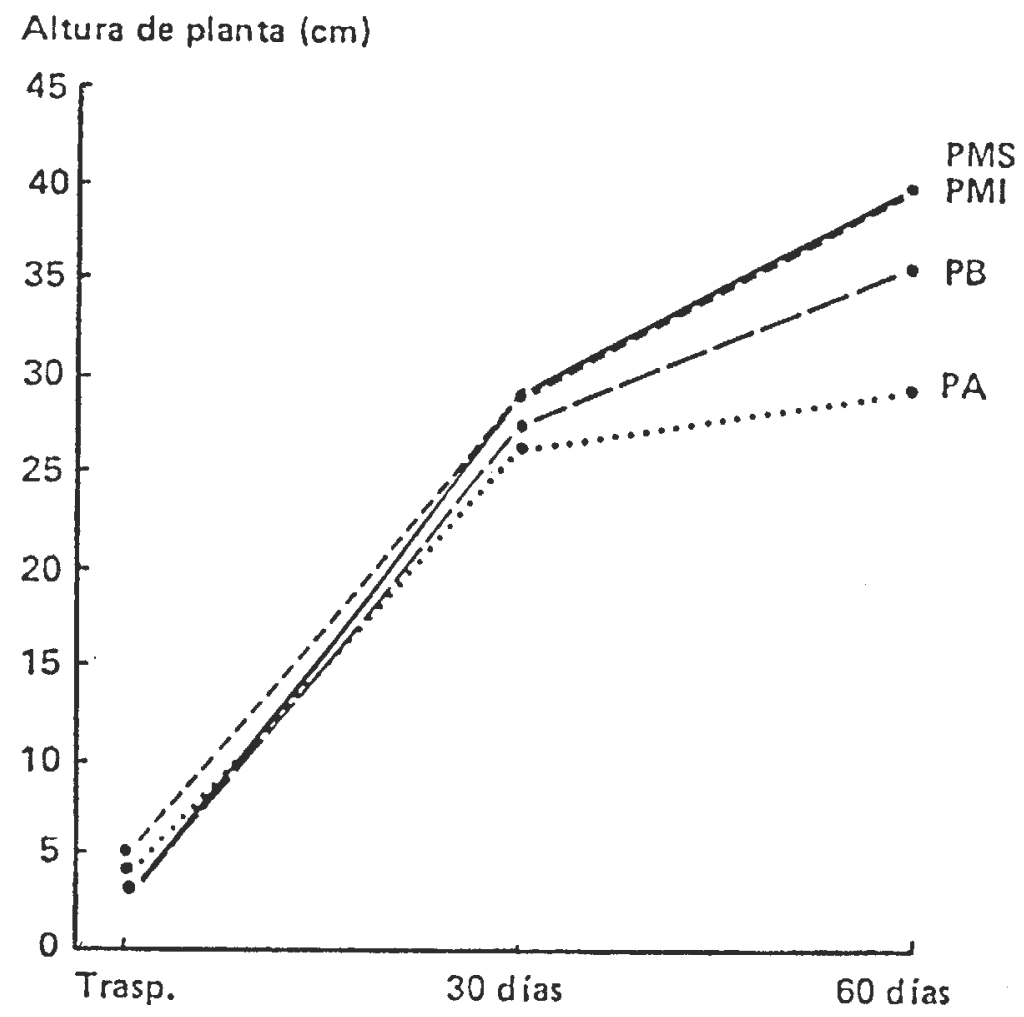

FIGURA 1. Evaluación de altura de plantas según la edad de los esquejes.

A los 30 días se observó una diferencia significativa entre tratamientos y además un gradiente de 3 a $5 \mathrm{~cm}$ de altura. Las PA eran más pequeñas que las PMS, PMI y PB. A los 60 días se mantiene esta relación y se notó una diferencia altamente significativa entre tratamientos.

\section{Producción de Tubérculos por Maceta}

En cuanto al número de tubérculos por macetas se observó una diferencia altamente significativa por tratamiento, ocupando el siguiente orden: PA, PMS, PB y PMI. En el tratamiento PA se tiene un incremento de $1 \mathrm{tub} /$ maceta. Respecto al peso total de los tubérculos por maceta no se observó diferencias entre tratamientos (Tabla 2, Figura 2).

\section{DISCUSIÓN Y CONCLUSIONES}

- Son importantes las diferencias de altura de planta encontradas a los 30 y 60 días, cuando se quiere utilizar la parte aérea de la misma para la producción de un mayor número de nuevos esquejes. Los tratamientos PMS y PMI presentaron un desarrollo más uniforme con respecto a los otros. 
TABLA 2. Producción de tubérculos por maceta, según el tipo de esquejes.

\begin{tabular}{lccc}
\hline Tratamiento & No. tub/maceta & $\begin{array}{c}\text { Peso tub/maceta } \\
\text { (g) }\end{array}$ & $\begin{array}{c}\text { Peso } \\
\text { promedio de } \\
\text { tub. }\end{array}$ \\
\hline Parte apical & $4.9(\mathrm{a})$ & $78.5(\mathrm{a})$ & $17.0(\mathrm{~b})$ \\
Parte media & $4.0(\mathrm{ab})$ & $82.2(\mathrm{a})$ & $23.8(\mathrm{a})$ \\
Parte media & $3.7(\mathrm{~b})$ & $71.3(\mathrm{a})$ & $21.6(\mathrm{ab})$ \\
Parte basal & $3.9(\mathrm{~b})$ & $72.0(\mathrm{a})$ & $19.6(\mathrm{ab})$ \\
\hline $\mathrm{n}=30$ & $\mathrm{CV}=32.5 \%$ & $\mathrm{CV}=22.8 \%$ & $\mathrm{CV}=41.1 \%$ \\
& (ALST=0.90) & (ALST $=11.67)$ & (ALST=5.7)
\end{tabular}

Los tratamientos seguidos con la misma letra no difieren significativamente al 5\% de probabilidad.
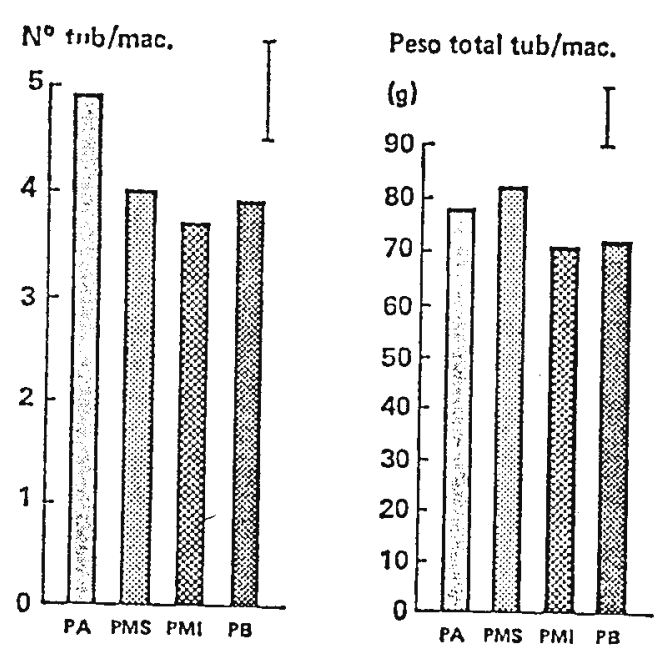

Pesa $\bar{x} /$ tub

(g)

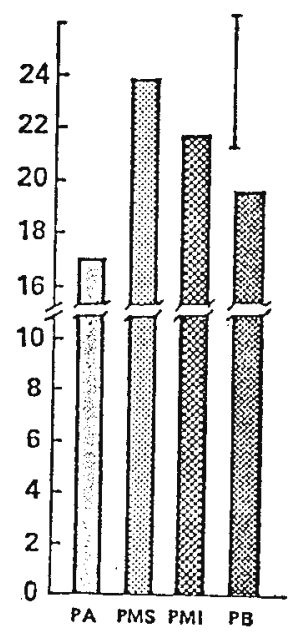

FIGURA 2. Peso total de tubérculos según el tipo de esquejes.

- El desarrollo de la planta en el tratamiento PA es más pequeño que las demás, pero, tiene la ventaja que la maduración es más temprana, ganándose aproximadamente 20 días en precocidad con relación a PMS, PMI y PB. 
- El número de tubérculos es uno de los factores de suma importancia en la producción de semilla básica. El rendimiento de las plantas del tratamiento PA produjo un $32 \%$ más en número de tubérculos cosechados por maceta, que el tratamiento PMI. Le siguieron los tratamientos PMS (8.1\%) y PB (5.4\%). Además como se mencionó tiene la ventaja de una mayor precocidad.

- El incremento del número de tubérculos/maceta puede estar referido a que los esquejes de PA corresponden a un material más joven en comparación con los otros esquejes.

- El rendimiento en cuanto al peso total de los tubérculos, provenientes de las plántulas obtenidas del tratamiento PMS fue mayor que PA, PB y PMI. Sin embargo, no fue significativo entre tratamientos. Se observó además un mayor desarrollo de la planta pero no se midió el área foliar específicamente.

- El peso promedio de los tubérculos indica un tamaño apropiado para siembras en el campo. El tratamiento PMS fue superior en un $40 \%$ con respecto a PA. Le siguieron los tratamientos PMI (27\%) y $\mathrm{PB}(15.3 \%)$.

- Se recomienda la utilización de esquejes de punta apical (PA) para la variedad ensayada, por su precocidad a la cosecha y mayor número de tubérculos-

- Para la producción de esquejes de la misma planta se recomienda el uso de los tratamientos PMS y PMI como plantas madres, por el buen desarrollo del área foliar.

\section{REFERENCIAS BIBLIOGRÁFICAS}

1. Bryan, J. E. Esquemas de multiplicación de semilla. Centro Internacional de la Papa. Lima, CIP, 6p.

2. Bryan, J. E. 1981. Rapid Multiplication techniques for potatoes, International Potato Center (CIP) Lima, 20p.

3. Centro Internacional de la Papa. 1975, Lima (Informe Anual)

4. Garay, A. Multiplicación de semilla por esqueje, 1976. Centro Internacional de la Papa, Lima, CIP. 11 p.

5. Meléndez, G. N.; Quevedo, B. M. 1980. Técnicas de multiplicación rápida de papa. Centro Internacional de la Papa. Lima, CIP. 43 p. 